\title{
Survival after ultrasonographic demonstration of portal venous gas due to mesenteric artery occlusion
}

\author{
Ronald A. Bloom ${ }^{1}$, Pinchus D. Lebensart ${ }^{1}$, Pavel Levy², Eli Craciun', Hiam \\ Anner ${ }^{2}$ and Jonah Manny ${ }^{2}$
}

\author{
Departments of ${ }^{1}$ Radiology and ${ }^{2}$ Surgery, Hadassah University Hospital, Ein Kerem, Jerusalem, Israel
}

\begin{abstract}
Summary: Portal vein gas as a result of bowel necrosis following occlusion of the superior mesenteric artery has an extremely grave prognosis. Only two previous cases have been recorded as having survived. In both those cases the diagnosis of portal vein gas was made on an abdominal radiograph. The present report is of the first such case surviving after ultrasonic demonstration of portal gas. In this case, no evidence of portal gas was seen on the abdominal radiograph. The increased sensitivity of ultrasound over plain radiography mandates urgent liver and portal ultrasound in all cases of suspected mesenteric event.
\end{abstract}

\section{Introduction}

The radiological demonstration of gas in the portal venous system is rare, some 150 cases having been reported. ${ }^{1}$ It is generally a lethal prognostic sign although it has occasionally been seen in benign conditions. ${ }^{2}$ The radiological demonstration of portal venous gas following mesenteric arterial occlusion is of particularly lethal import, only two cases having been recorded as surviving that event. $^{2,3}$

We recently reported the first case of portal venous gas shown by ultrasound in a patient with bowel necrosis due to mesenteric arterial occlusion. That patient died a month post-operatively. ${ }^{4}$ The present report is of a further case of hepatic portal venous gas due to this cause. In this case the patient survived after extensive small bowel resection. In neither of these two cases was portal gas seen in the plain abdominal radiograph.

\section{Case report}

A 72 year old man developed sudden lower abdominal pain which become worse overnight. On examination, he had right sided peritoneal irritation but no other significant clinical signs. There was a past history of intermittent claudication and coronary artery disease.

On ultrasound examination, bright reflective linear echoes were seen in the liver both centrally and peripherally with similar bright echoes seen rushing into the portal vein. The gallbladder wall was highly echogenic but did not have the reflective

Correspondence: R.A. Bloom, M.D.

Accepted: 4 July 1989 quality of gas shadows (Figures 1 and 2). A plain abdominal radiograph taken immediately after the ultrasound examination demonstrated a porcelain gallbladder, but no evidence of portal venous gas. A diagnosis of probable necrotic bowel was made and the patient was taken to surgery 24 hours after the onset of abdominal pain.

At operation extensive necrotic bowel was seen. No pulsations of the superior mesenteric artery were palpated distal to the origin of the ileo-colic artery. Resection of $120 \mathrm{~cm}$ of necrotic small bowel and ascending colon was performed and an end-toend anastomosis performed. The patient made an uneventful recovery.

\section{Discussion}

Portal venous gas as a sequel of intestinal gangrene following superior mesenteric artery occlusion is of dire prognostic significance. Liebman et al. in their review of the literature on portal venous gas in adults, collected 14 cases secondary to mesenteric artery occlusion. Of these only one survived and only a single further example has been reported. ${ }^{2,3}$ Portal venous gas is generally of poor prognostic significance in adults, with an overall survival rate of $25 \%$. This reflects the association with ischaemic necrotic bowel in most reported cases; in those cases mortality exceeds $90 \%$. $^{2,5}$ A much lower mortality is associated with non-ischaemic causes of portal venous gas, such as intra-abdominal sepsis, bowel distension, pancreatitis, gastric ulcer, and diverticulitis, ${ }^{2,6}$ and there are benign causes such as portal gas following barium enema examination. ${ }^{7}$

There is controversy as to the source of portal 


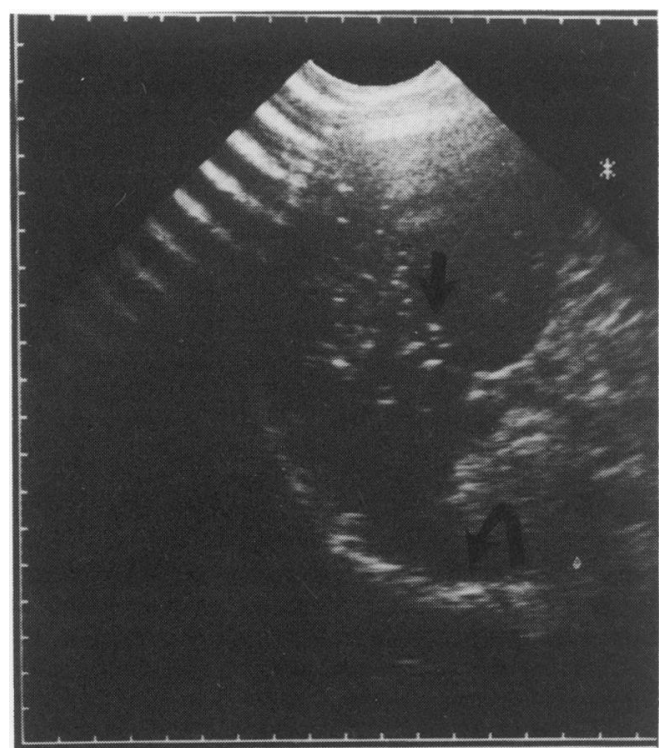

Figure 1 Bright reflective short linear shadows situated peripherally in the liver (straight arrow), indicating gas in portal vein branches. The curved arrow points to the diaphragm.

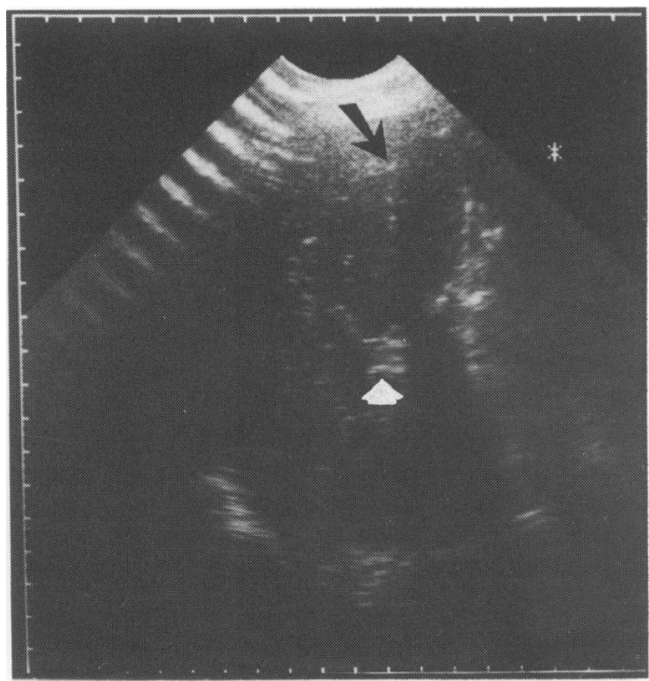

Figure 2 Gas shadows in the main portal vein (open arrowhead). The echogenic gallbladder wall is due to a porcelain gallbladder (oblique arrow).

\section{References}

1. Griffiths, D.M. \& Gough, M.H. Gas in the hepatic portal veins. Br J Surg 1986, 73: 172-176.

2. Leibmann, P.R., Patten, M.T., Manny, J., Benfield, J.R. \& Hechtman. H.B. Hepato-portal gas in adults : etiology, pathophysiology, and clinical significance. Ann Surg 1978, 187: 281-287. venous gas following superior mesenteric arterial occlusion. It is probably due to mucosal damage following intestinal infarction. ${ }^{2}$ The ultrasonic demonstration of portal venous gas is made by the demonstration of bright reflective echoes in the portal vein and its branches, and the diagnosis is made more certain by visualizing the centrifugal movement of high amplitude echoes within the portal veins (Figures 1 and 2).

In patients who develop portal vein gas as a sequel of vascular insult to the bowel, it is assumed that micro-bubbles form previous to the radiological appearance. ${ }^{8}$ These may initiate protein denaturation in the portal sinusoids with slowing of blood flow. The subsequent formation of larger bubbles may then partially or completely abolish portal blood flow reducing or preventing hepatic detoxification of blood borne toxins and bacteria, leading to toxic shock. ${ }^{9}$

It must be stressed that the picture of bowel necrosis is often subtle and the patient may exhibit few signs until overwhelming infection sets in. This is the reason for the almost $100 \%$ mortality rate when infection progresses to the point where gas is seen in the portal system following mesenteric artery obstruction. The earliest possible demonstration of portal vein gas is thus vital in trying to reduce the high mortality rate of bowel necrosis following obstruction to the superior mesenteric artery.

The increased sensitivity of ultrasound over plain radiography in revealing the presence of portal vein gas has been shown. ${ }^{4,10}$ In many patients with bowel necrosis there is considerable ileus and the liver cannot be adequately seen via the subcostal approach. In such cases it can always be examined through the intercostal spaces. In the present case the portal gas seen by ultrasound was not visible on an abdominal radiograph taken immediately afterwards. This demonstration of portal venous gas led to immediate laparotomy. As vascular impairment of the bowel had not been previously suspected, the subsequent rapid resection of necrotic bowel in our case may well have contributed to the successful outcome.

We recommend the performance of an ultrasound examination of the abdomen as an emergency test in all cases of suspected mesenteric event. The presence of portal vein gas in such a case mandates immediate laparotomy even in the presence of a normal abdominal radiograph.

3. Kranendonk, S.E., Bruining, H.A. \& Van Erk, H. Survival after portal venous gas due to mesenteric vascular occlusion. Br J Surg 1983, 70: 183-186. 
4. Bloom, R.A., Craciun, E., Jurim, O. \& Lebensart, P.D. The sonographic demonstration of hepatic venous gas in mesenteric arterial thrombosis. J Clin Gastroenterol 1988, 10: 226-228.

5. Traverso, L.W. Is hepatic portal venous gas an indication for exploratory laparotomy? Arch Surg 1982, 116: 936-938.

6. Cambria, R.P. \& Margolies, M.N. Hepatic portal venous gas in diverticulitis. Arch Surg 1974, 117: 834-835.

7. Stein, M.G., Crues, J.V. \& Hamlin, J.A. Portal venous gas associated with barium enema. Am J Radiol 1983, 140: 1171-1172.
8. Malm, S.W., Bhutani, V.K., Richie, W.W., Hall, M.L. \& Paul, D. Echogenic intravascular and hepatic micro-bubbles associated with necrotising enterocolitis. J Pediatr 1983, 103: 637-640.

9. Laing, F.C., Rego, J.D. \& Jeffrey, R.B. Ultrasonographic identification of portal vein gas. J Clin Ultrasound 1984, 19: 512-514. 